\title{
ELETROCATÁLISE DAS REAÇÕES DE OXIDAÇÃO DE HIDROGÊNIO E DE REDUÇÃO DE OXIGÊNIO
}

\author{
Edson A. Ticianelli*, Giuseppe A. Camara e Luís G. R. A. Santos \\ Instituto de Química de São Carlos, Universidade de São Paulo, CP 780, 13560-970 São Carlos - SP
}

Recebido em 6/7/04; aceito em 3/11/04; publicado na web em 28/2/05

\begin{abstract}
ELECTROCATALYSIS OF THE HYDROGEN OXIDATION AND OXYGEN REDUCTION REACTIONS. This work discusses the electrocatalytic processes taking place in the polymer electrolyte fuel cell electrodes, specifically the hydrogen oxidation reaction (HOR) and the oxygen reduction reaction (ORR), because these are clear examples of electrochemical reactions favored by the use of electrocatalysts. Since the gaseous reactants are very little soluble in the electrolyte, the use of special electrodes, named gas diffusion electrodes, is required to promote easy and continuous access of reactant gases to the electrocatalytic sites. Besides this, other important aspects such as the use of spectroscopic techniques and of theoretical models to improve the knowledge of the electrocatalytic systems are shortly discussed.
\end{abstract}

Keywords: electrocatalysis; hydrogen oxidation reaction; oxygen reduction reaction.

\section{INTRODUÇÃOO}

O termo Eletrocatálise começou a ser difundido a partir da década de 60, na discussão da influência do material do eletrodo sobre a cinética da reação eletroquímica de desprendimento de hidrogênio. Nos dias de hoje, o uso do termo tornou-se muito mais abrangente, referindo-se genericamente ao efeito da natureza do material do eletrodo sobre a cinética de qualquer reação eletroquímica. Na maioria dos casos, o eletrodo não é transformado durante a ocorrência da reação, servindo apenas como um aceitador ou doador de elétrons e participando do processo através de etapas de adsorção de reagentes e/ou intermediários.

Neste contexto, verifica-se que os fundamentos que governam as propriedades eletrocatalíticas têm muito em comum com aqueles da catálise heterogênea. Assim, diversos aspectos, muitos deles comuns à catálise heterogênea, governam os processos eletrocatalíticos: (1) a atividade eletrocatalítica é função da estrutura eletrônica dos átomos da superfície do material que compõe o eletrodo e (2) as propriedades superficiais, químicas e estruturais são fundamentais na definição da velocidade e do caminho da reação. Específico da eletrocatálise é o fato de que nos processos eletroquímicos estão sempre envolvidas reações de transferência de carga, sendo possível modificar a energia de ativação e/ou as propriedades da superfície ativa através do controle do potencial do eletrodo.

Inúmeras reações eletroquímicas com potencial tecnológico somente são viabilizadas quando realizadas sob condições que garantam uma velocidade (ou corrente elétrica) adequada, ou seja, com uso de materiais eletródicos que apresentem elevada eficiência eletrocatalítica. Em sistemas operacionais, fatores associados ao transporte de massa podem também se tornar etapas importantes no processo global de transferência de carga e, assim, a extensão da área superficial desempenha um papel crucial sobre sua velocidade. Neste trabalho são discutidos os processos eletrocatalíticos que ocorrem nas células a combustível com eletrólito polimérico (PEMFC), ou seja, as reações de oxidação de hidrogênio $(\mathrm{ROH})$ e de redução de oxigênio (RRO). Estas são exemplos claros de reações eletroquímicas viabilizadas pelo uso de eletrocatalisadores. Como se trata de reagentes gasosos muito pouco solúveis é tam-

*e-mail: edsont@iqsc.usp.br bém necessário o uso de eletrodos especiais para que se possa promover o acesso contínuo dos gases aos sítios eletrocatalíticos, sendo estes detalhes também abordados nas análises efetuadas.

\section{BREVE DESCRIÇÃO DA TECNOLOGIA DAS CÉLULAS A COMBUSTÍVEL}

Atualmente, uma das alternativas mais promissoras para aplicação como fonte de energia elétrica em sistemas estacionários, portáteis e móveis (veículos) são as células a combustível, particularmente aquelas que são alimentadas por hidrogênio/ar. Estes dispositivos são capazes de converter energia química armazenada nos reagentes em energia elétrica e calor, através de um par de reações eletroquímicas acopladas, de maneira mais ecológica e eficiente, sendo apresentado na Figura 1 um esquema que representa seus princípios de operação.

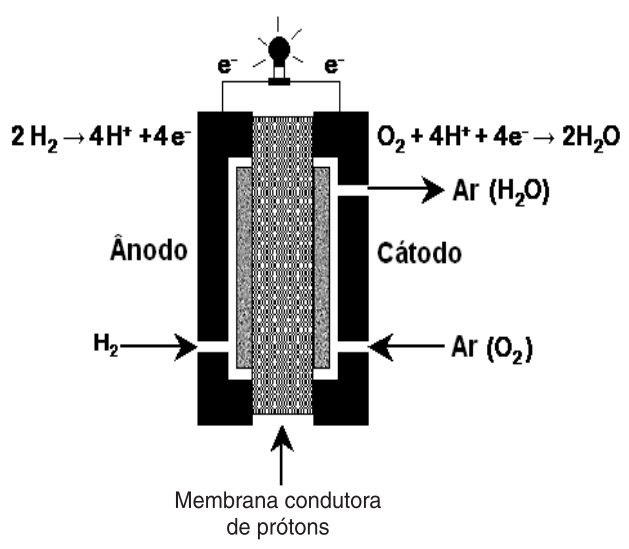

Figura 1. Esquema de operação de uma célula a combustível do tipo PEMFC

No atual estágio de desenvolvimento existem vários tipos de células a combustível, que são classificadas de acordo com o eletrólito utilizado e que se prestam a diferentes tipos de aplicações. Este assunto tem sido discutido em detalhes em publicações prévias ${ }^{1-3}$, sendo aqui feita apenas uma descrição breve das principais características das diferentes tecnologias: 
Alcalinas (AFC): utilizam $\mathrm{KOH}$ 6,0 M como eletrólito e operam em temperaturas de até $120{ }^{\circ} \mathrm{C}$, sendo adequadas para aplicações onde se dispõe de hidrogênio puro e se requer alta densidade de potência.

Eletrólito polimérico (PEMFC): são montadas usando uma membrana de troca protônica como eletrólito, sendo suas características similares às da célula alcalina, com a vantagem de não exigirem combustível puro e com a desvantagem de exigirem metais nobres nos eletrodos.

Ácido fosfórico (PAFC): utilizam ácido fosfórico concentrado $(98 \%)$ como eletrólito e operam em temperaturas da ordem de $200{ }^{\circ} \mathrm{C}$, sendo mais adequadas para aplicações estacionárias.

Carbonato fundido (MCFC): o eletrólito é uma mistura de carbonato de lítio e potássio fundidos. Operam em temperaturas da ordem de $600{ }^{\circ} \mathrm{C}$, sendo mais adequadas para as aplicações estacionárias em larga escala (centenas de $\mathrm{kW}$ ).

Óxidos sólidos (SOFC): usam uma cerâmica de zircônio estabilizada com ítria como eletrólito, operam em temperaturas da ordem de $1000{ }^{\circ} \mathrm{C}$. Muitos destes sistemas também se destinam a aplicações estacionárias em larga escala (centenas de kW), mas existem projetos que visam o desenvolvimento de módulos com potência da ordem de $1 \mathrm{~kW}$.

Existe atualmente um grande interesse em promover aplicações de células a combustível como sistemas autônomos de geração de energia elétrica. Em particular, sistemas de pequeno porte (1-20 kW) funcionando com hidrogênio reformado e ar, encontram suas aplicações em residências, na geração de eletricidade em localidades remotas, em veículos elétricos híbridos associados a baterias secundárias, em veículos de pequeno porte como fonte única de energia elétrica e em diversos outros usos. Unidades maiores (150-250 kW) podem ser usadas em veículos elétricos de grande porte (ônibus) ou no fornecimento de energia elétrica doméstica para conjuntos integrados (hospitais, shopping centers, etc.) e para localidades remotas. Unidades ainda maiores (250 kW-20 MW) têm sido consideradas para aplicações como "load leveling", cogeração, aproveitamento de rejeitos industriais, etc. Uma dezena de outras possibilidades de aplicações em pequenos sistemas (40W a $1 \mathrm{~kW}$ ) tem também aparecido nos últimos anos, que incluem microcomputadores portáteis, telefones celulares, etc.

\section{BREVE DESCRIÇÃO DOS ELETRODOS DE DIFUSÃO DE GÁS}

Devido à baixa solubilidade dos gases reagentes (hidrogênio e oxigênio), para o bom funcionamento das células a combustível, é indispensável o uso dos eletrodos de difusão de gás (EDG), que são eletrodos com alta área ativa onde ocorre a difusão na fase gasosa dos reagentes para alcançar o sítio eletrocatalítico. O desenvolvimento desses eletrodos, em particular do cátodo de oxigênio, é de extremo interesse visto que também apresenta aplicações em baterias de metal/ar e na indústria de cloro/soda.

Embora diversos tipos de eletrodos de difusão de gás tenham sido estudados nos últimos 30 anos, os eletrodos hidrofóbicos de carbono poroso catalisado com platina pura ou em ligas são os que têm apresentado maior eficiência para aplicação em células a combustível com eletrólitos aquosos ácidos, alcalinos e com eletrólito de membrana polimérica. Neste último caso, uma limitação encontrada no passado para o uso destes eletrodos era a elevada carga de platina requerida para obter desempenho satisfatório. Entretanto, no final da década de 80 foi demonstrado ${ }^{4-6}$ que estas células podem operar com eletrodos contendo baixas cargas de catalisador, em níveis de $0,2 \mathrm{mgPt} / \mathrm{cm}^{2}$. Em função deste fato houve um aumento acentuado do interesse nesta tecnologia sendo que, atual- mente, mais de uma centena de grupos têm realizado pesquisas neste sistema.

O desempenho das células a combustível é limitado por perdas relacionadas à membrana, aos eletrodos e ao sistema eletro/eletrônico. Perdas significativas são originadas de: i) cinética reacional limitada, em particular da reação de redução de oxigênio; ii) envenenamento do catalisador anódico, particularmente quando se usa hidrogênio produzido por reforma; iii) condutividade protônica limitada na camada catalisadora dos eletrodos; iv) permeabilidade efetiva limitada do oxigênio ou hidrogênio na camada catalisadora do eletrodo e v) limitação difusional do gás na camada difusora do eletrodo. Tendo em vista o objetivo deste trabalho, a seguir serão discutidos os aspectos mais importantes dos fenômenos (i) e (ii) acima relacionados.

\section{ELETROCATÁLISE DA REAÇÃO DE REDUÇÃO DE OXIGÊNIO}

A reação de redução de oxigênio (RRO) é considerada como uma das reações eletrocatalíticas mais importantes por causa da sua função em conversores eletroquímicos de energia, vários processos industriais e em corrosão. Conseqüentemente, há muitos anos é foco de interesse das pesquisas eletroquímicas. A redução de oxigênio continua a ser um desafio para eletroquímicos devido à sua complexidade cinética e à necessidade de melhores eletrocatalisadores.

A redução de oxigênio é uma reação multieletrônica que usualmente inclui várias etapas elementares no mecanismo reacional. A RRO em soluções aquosas ácidas ocorre segundo dois mecanismos globais clássicos já conhecidos ${ }^{7,8}$ :

I) Mecanismo direto ou mecanismo 4-elétrons:

$\mathrm{O}_{2}+4 \mathrm{H}^{+}+4 \mathrm{e}^{-} \rightarrow 2 \mathrm{H}_{2} \mathrm{O} \quad \mathrm{E}^{\circ}=1,229 \mathrm{~V}$

II) Mecanismo peróxido ou mecanismo 2-elétrons:

$\mathrm{O}_{2}+2 \mathrm{H}^{+}+2 \mathrm{e}^{-} \rightarrow \mathrm{H}_{2} \mathrm{O}_{2} \quad \mathrm{E}^{\circ}=0,670 \mathrm{~V}$

sendo que o peróxido de hidrogênio pode ser reduzido em uma etapa posterior:

$\mathrm{H}_{2} \mathrm{O}_{2}+2 \mathrm{H}^{+}+2 \mathrm{e}^{-} \rightarrow 2 \mathrm{H}_{2} \mathrm{O} \quad \mathrm{E}^{o}=1,770 \mathrm{~V}$

ou sofrer decomposição química segundo:

$2 \mathrm{H}_{2} \mathrm{O}_{2} \rightarrow 2 \mathrm{H}_{2} \mathrm{O}+\mathrm{O}_{2}$

$\mathrm{O}$ potencial $\mathrm{E}^{\circ}$ nestas equações corresponde ao valor padrão da reação em função do eletrodo reversível de hidrogênio (ERH) a $25{ }^{\circ} \mathrm{C}$. A distinção entre estes dois mecanismos é dificultada pelo fato de que a redução direta também pode envolver a formação de peróxido, desde que este permaneça adsorvido na superfície do eletrodo sem sofrer dessorção ou decomposição, e a sua redução ocorra subseqüentemente. Sendo este processo também de 4 elétrons, porém, chamado de mecanismo em série. Em potenciais mais catódicos juntamente com o mecanismo via 4 elétrons ocorre o mecanismo via 2 elétrons, sendo chamado de mecanismo paralelo. Em geral, a técnica de eletrodos de disco-anel rotatório é a mais utilizada na verificação e diferenciação entre os dois mecanismos operacionais que regem a reação de redução da molécula de $\mathrm{O}_{2}$, sendo que nesta técnica o eletrodo de anel é utilizado somente para monitorar a produção de peróxido oriunda do eletrodo de disco ${ }^{7,8}$. 
O mecanismo direto e o mecanismo em série são indistinguíveis pelo método do eletrodo de disco-anel rotatório. A redução de oxigênio procede quantitativamente através do mecanismo peróxido em um grande número de superfícies eletródicas, particularmente em meio alcalino.

Nas últimas décadas, muitos esforços têm sido dedicados ao estudo do mecanismo da RRO em soluções aquosas ácidas e alcalinas sobre diferentes materiais de eletrodo ${ }^{9-23}$. Em eletrólitos ácidos, platina e ligas de platina ainda são consideradas as melhores opções para a redução de oxigênio, tanto em termos dos mais baixos sobrepotenciais desejáveis para promover a reação, quanto da estabilidade requerida. Assim, os eletrocatalisadores baseados em platina são necessários para prover estabilidade no ambiente corrosivo da PEMFC e, neste sentido, as ligas de platina favorecem a atividade catalítica desejada ao mesmo tempo em que permitem a diminuição do teor de $\mathrm{Pt}$, com conseqüente ganho econômico.

Sobre platina e metais da família da platina ocorre o mecanismo paralelo, mas a predominância é do mecanismo direto via 4 elétrons. Para estes metais há duas propostas para a primeira etapa reacional. A primeira proposta é de Damjanovic e colaboradores ${ }^{24}$, em que a transferência de prótons ocorre simultaneamente com a transferência de carga. A segunda visão é de que o mecanismo mais comum, a redução via 4 elétrons sobre Pt, envolve adsorção química seguida de dissociação da molécula de $\mathrm{O}_{2}$ sobre a superfície de platina que, provavelmente, ocorre simultaneamente com a transferência de $\operatorname{carga}^{25}$.

Ligas como Pt-Co, Pt-Cr, Pt-Ti, Pt-V e Pt-Fe suportadas em carbono $(\mathrm{Pt}-\mathrm{M} / \mathrm{C})$ têm sido muito estudadas para a redução de oxigênio em meio ácido. Alguns estudos sobre estas ligas têm mostrado um aumento da atividade eletrocatalítica para a RRO em relação à Pt pura ${ }^{10,25,26}$. Jalan e Taylor ${ }^{26}$ estudaram a reação de redução de oxigênio em diversas ligas de platina suportadas em carbono (Pt-Cr, Pt-V, Pt-Ti e Pt-Al) em ácido fosfórico e propuseram que a melhora da atividade catalítica da platina resulta do encurtamento da distância interatômica Pt-Pt nas ligas quando comparadas à Pt pura. A mesma interpretação foi dada por Appleby ${ }^{27}$ e por Mukerjee e Srinivasan ${ }^{28}$. Entretanto, Glass e colaboradores ${ }^{29}$ consideraram $^{2}$ que a melhora é específica para ligas catalíticas altamente dispersas, porque o efeito não foi observado para macro aglomerados de ligas de Pt-Cr. Uma análise da RRO em ligas de Pt-Cr em PAFCs mostrou um aumento na área superficial depois de prolongada operação, devido à dissolução do metal não nobre ${ }^{30}$.

Ligas de Pt-Co e Pt-Co-Cr suportadas em carbono podem aliviar apreciavelmente o sobrepotencial da redução de oxigênio em relação à platina pura ${ }^{31,32}$. Ligas binárias de Pt-Fe exibem melhor resistência à sinterização das partículas a elevadas temperaturas que catalisadores que contêm platina pura. Este efeito é chamado de "efeito âncora" do ferro para a platina sobre substrato de carbono. A mobilidade dos átomos de platina sobre substrato de carbono é dificultada quando o ferro está presente. Aricò e colaboradores ${ }^{33}$ realizaram estudos de difração de raios X para Pt, Pt-Co e Pt-Co$\mathrm{Cr}$ e os resultados mostraram que a Pt suportada em carbono tem estrutura cúbica de face centrada, porém, Pt-Co e Pt-Co-Cr suportadas em carbono têm estrutura de face centrada tetragonal, o que está de acordo com a proposta de Glass ${ }^{29}$. Outros estudos em ligas de Pt-Co suportadas em carbono mostraram que uma estrutura cristalina desordenada é mais resistente à corrosão que uma ordenada e mantém alta a atividade catalítica deste material por um longo tempo ${ }^{17}$.

Fatores como a mudança na estrutura superficial e na energia de interação do oxigênio com o material da liga também podem alterar as propriedades eletrocatalíticas da platina nas ligas. $\mathrm{O}$ tratamento térmico exerce função primordial na obtenção de eletrocatalisadores à base de platina. Para dois catalisadores dispersos em carbono com a mesma composição e obtidos pelo mesmo método, diferentes tratamentos térmicos podem produzir atividades catalíticas completamente diferentes. O tratamento térmico permite controlar o tamanho de partículas e limpar a superfície dos catalisadores de impurezas. Além disto, com o aumento da temperatura do tratamento térmico ocorre aumento do tamanho das partículas e, portanto, um decréscimo da área superficial. Pt pura e catalisadores formados por ligas de platina mostram aumento da atividade específica com o acréscimo do tamanho das partículas até aproximadamente $5 \mathrm{~nm}$, sendo isto decorrente de alterações na ocupação da banda-d da platina e de mudanças dos arranjos cristalinos dos átomos que compõem a superfície das nanopartículas.

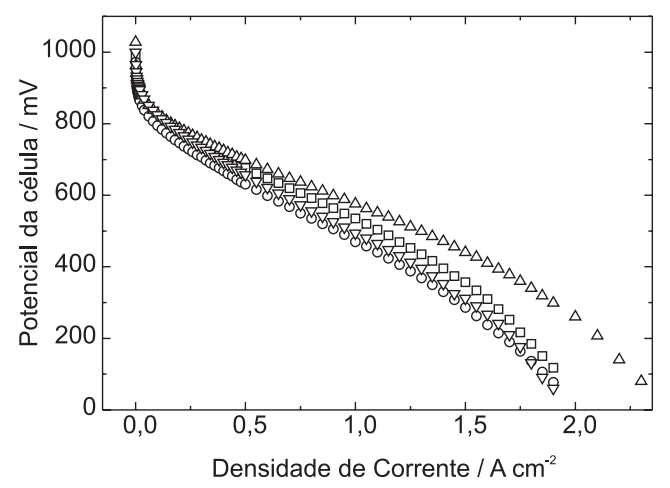

Figura 2. Curvas de polarização para uma célula do tipo PEMFC alimentada com $\mathrm{H}_{2} / \mathrm{O}_{2}$ com eletrocatalisadores de Pt/C e PtV/C para a RRO a $85^{\circ} \mathrm{C}$. Efeito da pressão de suprimento de $\mathrm{O}_{2}\left(P_{\mathrm{O}_{2}}\right)$, mantendo-se a pressão de hidrogênio constante em $1 \mathrm{~atm}$ : $(\square) P t / C, P_{O 2}=1 \mathrm{~atm}$; (O) PtV/C, $P_{O 2}=1$ atm; $(\triangle) P t / C, P_{O 2}=2 \mathrm{~atm} ;(\nabla) P t V / C, P_{O 2}=2 \mathrm{~atm}$. Ânodos com $0,4 \mathrm{mg} P t /$ $\mathrm{cm}^{2}$; cátodos com 0,4 mg Pt-V/cm². Reproduzido da ref. 34, com permissão da Elsevier Science

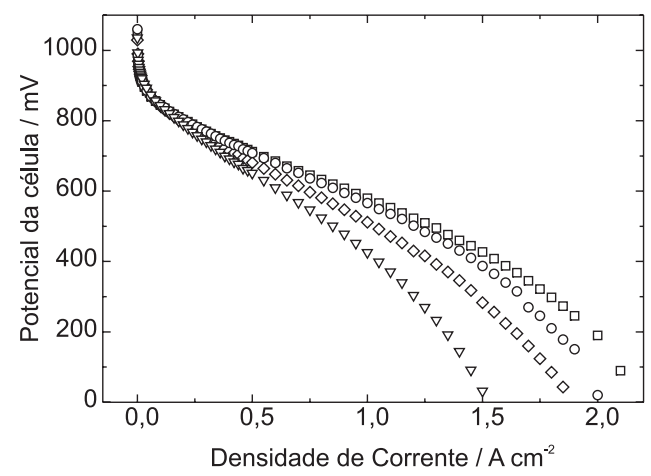

Figura 3. Curvas de polarização para uma célula do tipo PEMFC alimentada com $\mathrm{H}_{2} / \mathrm{O}_{2}$ com eletrocatalisadores de Pt/C e PtV/C para a RRO a $85^{\circ} \mathrm{C}$. Efeito da pressão de suprimento de $\mathrm{O}_{2}\left(P_{\mathrm{O}_{2}}\right)$, mantendo-se a pressão de hidrogênio constante em $1 \mathrm{~atm}$ : (O) $\mathrm{Pt} / \mathrm{C}, P_{O 2}=3 \mathrm{~atm} ;(\nabla) \mathrm{PtV} / \mathrm{C}, P_{O 2}=3$ atm; ( $\square$ ) Pt/C, $P_{O 2}=5 \mathrm{~atm} ;(\diamond) P t V / C, P_{O 2}=5 \mathrm{~atm}$. Ânodos com $0,4 \mathrm{mg} \mathrm{Pt} /$ $\mathrm{cm}^{2}$; cátodos com 0,4 mg Pt-V/cm². Reproduzido da ref. 34, com permissão da Elsevier Science

$\operatorname{Min}^{10}$ atribuiu o aumento da atividade catalítica da platina na liga Pt-V a uma combinação de efeitos eletrônicos (vacâncias na banda-d da platina) e geométricos (diminuição da distância Pt-Pt). As Figuras 2 e 3 ilustram alguns resultados obtidos em estudos sobre a RRO em células PEMFC unitárias com ligas Pt-V 1:1 à 
pressão de $1 \mathrm{~atm}$ de $\mathrm{O}_{2}$, onde se observa a baixa atividade catalítica nestas condições ${ }^{34}$. Entretanto, em pressões $\geq 2$ atm um aumento da propriedade eletrocatalítica de $\mathrm{Pt}-\mathrm{V} / \mathrm{C}$ comparado à $\mathrm{Pt} / \mathrm{C}$ é observado (comparar Figuras 2 e 3). Este resultado indica a ocorrência de um mecanismo eletrocatalítico para a RRO diferente em Pt$\mathrm{V} / \mathrm{C}$ e $\mathrm{Pt} / \mathrm{C}^{34}$. Faubert e colaboradores ${ }^{35}$ prepararam catalisadores para a $\mathrm{RRO}$ com $\mathrm{Fe}(\mathrm{OH})_{2} / \mathrm{C}$ pirolisado a temperaturas $\geq 600{ }^{\circ} \mathrm{C}$ e observaram corrente estável na célula a combustível quando o catalisador era preparado em temperaturas $\geq 800{ }^{\circ} \mathrm{C}$.

Apesar de todos estes esforços, os desafios da complexidade cinética da RRO e da necessidade de superação de uma barreira de energia de ativação significativa ainda permanecem e requerem o desenvolvimento de catalisadores alternativos eficientes e de baixo custo, associados com informações mais detalhadas dos mecanismos reacionais que ainda não são totalmente entendidos. O que se pode afirmar é que se trata de uma reação altamente irreversível em soluções aquosas, gerando perdas excessivas de voltagem e, portanto, limitando sua aplicação em sistemas eletroquímicos que utilizam o eletrodo de oxigênio, em particular as células a combustível.

\section{ELETROCATÁLISE DA REAÇÃO DE OXIDAÇÃO DE HIDROGÊNIO}

Um outro problema muito importante relacionado à eficiência das células a combustível está relacionado com a pureza do hidrogênio. Se o gás é produzido por eletrólise da água, a pureza é elevada e o catalisador de platina dispersa é plenamente satisfatório para promover com alta eficiência a reação de oxidação de hidrogênio $(\mathrm{ROH})$ que tem lugar no ânodo das células. Entretanto, se o hidrogênio é produzido através da reforma de outros combustíveis, (gás natural, biogás, metanol, etanol, etc.) o produto pode vir acompanhado de impurezas tais como $\mathrm{CO}$ e compostos de enxofre (em níveis de $0,1 \%$ ) que se adsorvem fortemente sobre a platina, diminuindo de forma drástica a atividade do eletrocatalisador em temperaturas abaixo de $150{ }^{\circ} \mathrm{C}$.

Através de estudos utilizando eletrodos de metais maciços, já se sabe que a presença de um segundo elemento além da platina, como $\mathrm{Ru}, \mathrm{Sn}, \mathrm{Os}, \mathrm{Mo}$, etc, formando ligas ou co-depósitos, leva a um incremento significativo na tolerância ao $\mathrm{CO}$ se comparado à platina pura ${ }^{10,36-39}$. A busca do entendimento do mecanismo de envenenamento tem se concentrado em uma variedade de abordagens experimentais ${ }^{36,40}$, incluindo o uso de técnicas eletroquímicas, UV-visível in situ, espectroscopia de infravermelho por reflectância, absorção de raios-X, etc. ${ }^{10,36-40}$.

Diversos estudos realizados a $25{ }^{\circ} \mathrm{C}$ em platina ou ligas de $\mathrm{Pt}$ dispersa em carbono indicam que o envenenamento ocorre devido à forte adsorção de $\mathrm{CO}$ na superfície do catalisador, que bloqueia a adsorção da espécie reagente (hidrogênio). Exemplos do forte efeito causado pela presença de $\mathrm{CO}$ na curvas de polarização de uma célula PEMFC são mostrados na Figura 4 para ânodos formados com Pt/C, Pt-Ru/C e Pt-Mo/C. No caso das ligas de Pt, um mecanismo bifuncional tem sido proposto para explicar a tolerância melhor em relação à presença do contaminante. De acordo com este modelo, a espécie reagente adsorve-se preferencialmente nos átomos de $\mathrm{Pt}$, enquanto que o outro metal $(\mathrm{Ru}, \mathrm{Mo}$, etc) produz espécies oxigenadas ou óxidos hidratados que atuam diretamente na oxidação do envenenador da reação. Nestas condições, a corrente origina-se apenas da oxidação do reagente que ocorre nas zonas da superfície da Pt livres de CO que se formam ao redor dos átomos do outro metal, sendo que o restante da área permanece coberto pela compacta monocamada de $\mathrm{CO}$ adsorvido.

Uma avaliação crítica dos resultados até agora obtidos permite concluir que o desenvolvimento de novos materiais e estruturas
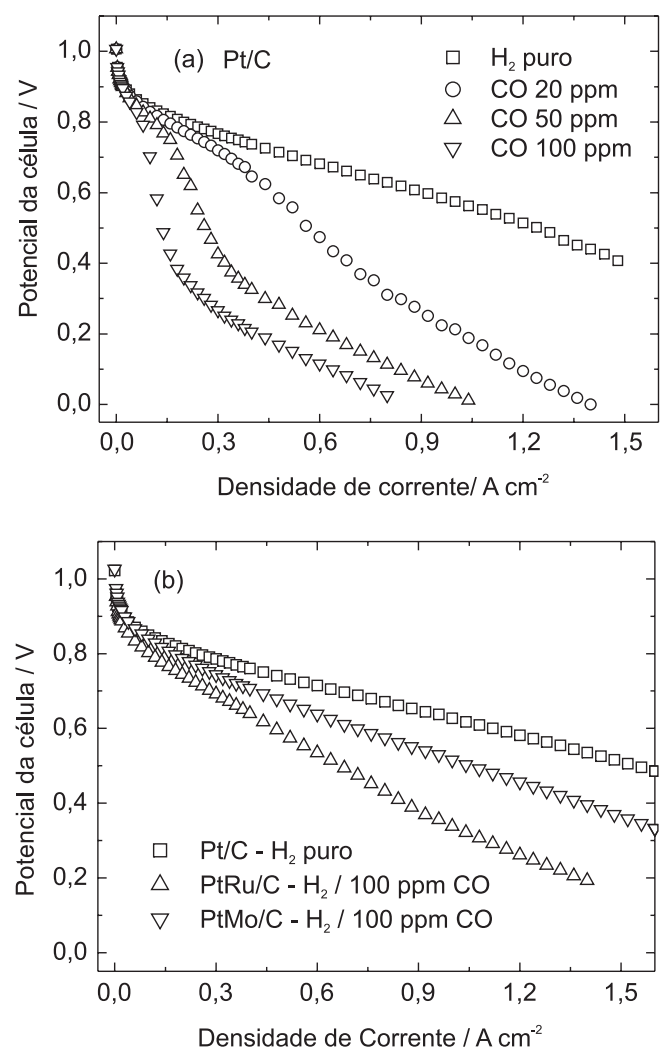

Figura 4.: Desempenho de células unitárias $\left(\mathrm{H}_{2} / \mathrm{O}_{2}\right)$ com diferentes catalisadores anódicos: (a) efeito do teor de CO no hidrogênio para o catalisador de Pt/C; (b) efeito do material eletrocatalisador para hidrogênio com 100 ppm de CO. Cátodos com 0,4 mg Pt/cm²; ânodos com 0,4 mg Pt-M/ $\mathrm{cm}^{2}$

eletródicas mais ativas para a $\mathrm{ROH}$ na presença de $\mathrm{CO}$ é ainda um campo muito aberto para pesquisas. Isto decorre do fato de que nem todos os fenômenos associados aos processos envolvidos estão plenamente caracterizados, sendo ainda requerida muita pesquisa de forma a superar as dificuldades envolvidas.

\section{CARACTERIZAÇÃO DOS MATERIAIS POR ESPECTROSCOPIA DE ABSORÇÃO DE RAIOS X}

Uma das causas da melhora na atividade eletrocatalítica de ligas de platina, tanto para a RRO como para a ROH (na presença de $\mathrm{CO}$ ), tem sido atribuída a modificações na estrutura eletrônica causadas pela presença do elemento adicional, que afeta tanto a cinética da transferência de carga como os processos de adsorção de oxigênio, hidrogênio ou monóxido de carbono. Investigações usando espectroscopia de absorção de raios-X (XAS) ${ }^{40-43}$ têm mostrado que é possível medir mudanças de natureza eletrônica (como vacâncias na banda 5-d da Pt) e nas interações atômicas de curto alcance (como a distância das ligações Pt-Pt e o número de coordenação) com especificidade elementar em condições in situ para vários tipos de sistemas eletroquímicos ${ }^{43}$.

A aplicação da técnica XANES ("X-absorption near-edge structure") a ligas nanocristalinas de Pt-Co, Pt-V, Pt-Ru, Pt-Sn e Pt-Os suportadas em carbono tem mostrado que a associação de tais espécies interfere tanto na estrutura atômica como eletrônica da platina ${ }^{40,43-48}$.

Exemplos de espectros XANES obtidos na borda $\mathrm{L}_{3}$ da Pt para alguns eletrocatalisadores dispersos de Pt, Pt-Ru e Pt-Os são mos- 


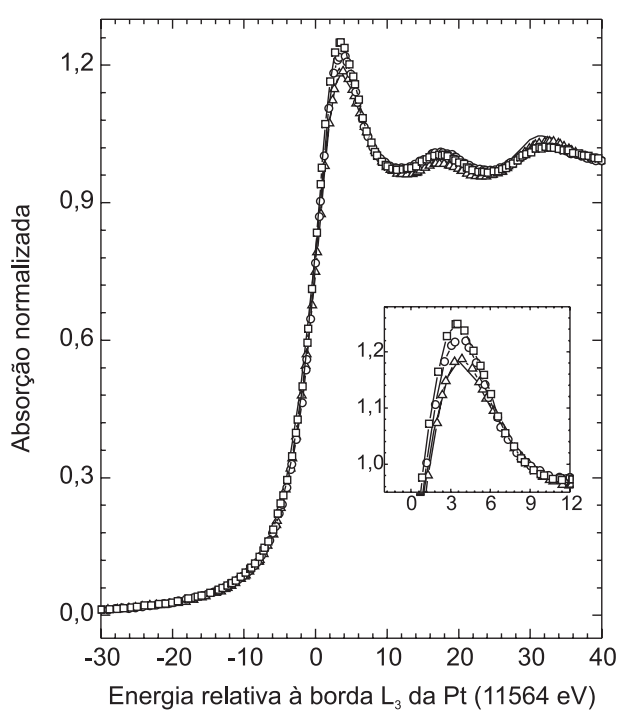

Figura 5. Espectros XANES na borda Pt-L . (-) Pt metálica; $(\triangle) P t / C$; (O) e ( $\square$ ) Pt-Ru/C preparados por dois procedimentos distintos. Todos os materiais dispersos em carbono foram analisados in situ, polarizados a $80 \mathrm{mV}$ vs. ERH. Reproduzido da ref. 43, com permissão da Elsevier Science

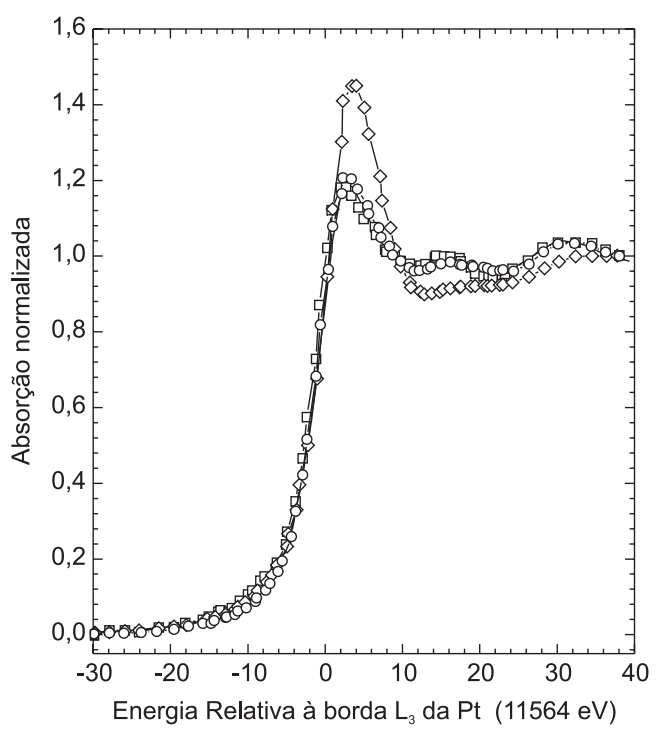

Figura 6. Espectros XANES na borda Pt- $L_{3^{*}}$ (-) Pt metálica; Os símbolos $(\triangle),(\diamond)$ e $(\mathrm{O})$ referem-se a catalisadores de PtOs/C preparados de formas distintas. Todos os materiais dispersos em carbono foram analisados in situ, polarizados a $80 \mathrm{mV}$ vs. ERH. Reproduzido da ref. 40, com permissão da Springer-Verrlag

trados nas Figuras 5 e 6 . Nota-se que a presença de $\mathrm{Ru}$ aumenta o pico de absorção de Raios X localizado em cerca de $5 \mathrm{eV}$, o que é uma evidência do esvaziamento da banda $5 \mathrm{~d}$ da $\mathrm{Pt}^{43-47}$. No caso do Os nenhum efeito é observado. Resultados como estes para outros sistemas têm indicado que a associação de $\mathrm{Sn}$, Co ou V à Pt promove o abaixamento energético das bandas $5 \mathrm{~d}$ da Pt e da fração vacância/átomo ${ }^{44-46}$. Estes efeitos são particularmente importantes do ponto de vista eletrocatalítico, uma vez que a criação de vacâncias no metal facilita a formação de espécies oxigenadas (oriundas da ativação de moléculas de $\mathrm{H}_{2} \mathrm{O}$ ), que são as principais responsáveis pela oxidação de $\mathrm{CO}$ a $\mathrm{CO}_{2}$.
Em um trabalho recente, Russel e colaboradores ${ }^{47}$ investigaram eletrocatalisadores de Pt-Ru e Pt-Mo dispersos em carbono através de experimentos eletroquímicos e de XAS. Estes autores concluiram que o mecanismo que facilita a oxidação de $\mathrm{CO}$ é diferente nos dois catalisadores. Para $\mathrm{PtRu} / \mathrm{C}$ a formação de uma liga diminuiria a força de quimissorção Pt-CO, enquanto que em $\mathrm{PtMo} / \mathrm{C}$ a melhora de desempenho eletroquímico (comparado à $\mathrm{Pt} / \mathrm{C}$ ) estaria atribuída a efeitos eletrônicos. Estes efeitos seriam os responsáveis pela redução dos valores do sobrepotencial de ativação, ocasionando a oxidação do $\mathrm{CO}$ em potenciais inferiores aos da $\mathrm{Pt} / \mathrm{C}$.

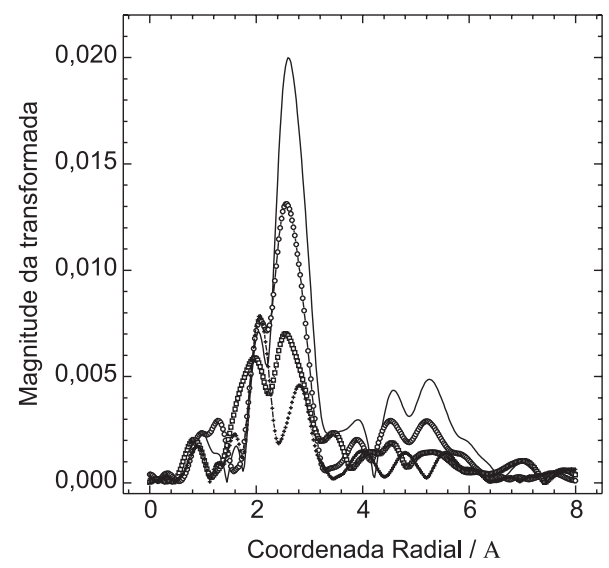

Figura 7. Transformadas de Fourier do sinal EXAFS para (-) Pt metálica; (O) Pt/C; ( $\square$ ) PtRu/C preparado conforme procedimento descrito na ref. 44; (+) Pt-Ru/C comercial. Todos os materiais dispersos em carbono foram analisados in situ, polarizados a $80 \mathrm{mV}$ vs. ERH. Reproduzida da ref. 43, com permissão da Elsevier Science

A técnica de EXAFS ("extended x-ray absorption fine structure)" tem sido também muito empregada na análise de catalisadores dispersos, em particular quando são requeridas informações sobre a coordenação e o ordenamento atômico de curta distância. Na Figura 7 apresentam-se exemplos dos resultados obtidos sob diversas condições experimentais na borda $\mathrm{L}_{3}$ da $\mathrm{Pt}$ para catalisadores de $\mathrm{Pt} / \mathrm{C}$ e $\mathrm{Pt}-\mathrm{Ru} / \mathrm{C}$. O desdobramento do pico da transformada de Fourier do sinal EXAFS da Pt nos materiais tratados termicamente é uma evidência clara da formação de uma liga verdadeira.

A interpretação dos resultados de EXAFS por modelamento atesta que a adição de $\mathrm{Sn}$ provoca um aumento da distância de ligação $\mathrm{Pt}^{-} \mathrm{Pt}^{46}$, enquanto que $\mathrm{Ru}$, Co e V levam a uma diminuição $0^{10,43}$. Estes efeitos implicam em uma mudança significativa nas características eletrocatalíticas destes materiais frente à RRO ou à $\mathrm{ROH}$ na presença de $\mathrm{CO}$, resultando em diferentes energias de ativação, ordens de reação, coberturas de superfície, etc. Neste contexto, as pesquisas envolvendo técnicas de caracterização física têm contribuído enormemente para a elucidação dos efeitos provocados pela inserção de outros metais à platina, no que se refere à atividade catalítica destes materiais frente a inúmeras reações eletrocatalíticas.

\section{MODELAMENTO E ELETROCATÁLISE}

Além das técnicas de caracterização física, o uso de modelos teóricos que consigam explicar e prever as propriedades catalíticas dos materiais é um ponto de interesse crescente em eletrocatálise. Nos últimos anos vêm sendo realizados vários estudos que tratam do aspecto teórico da reatividade de superfícies metálicas, com ênfase nos materiais baseados em $\mathrm{Pt}^{46-55}$. Usando modelos funda- 
mentados em formulações quânticas, tem sido possível separar os efeitos geométricos e eletrônicos que determinam as propriedades da adsorção. Baseando-se em simulações quânticas do tipo Monte Carlo, Norskov e colaboradores ${ }^{53-55}$ têm investigado vários dos fatores que interferem na atividade de superfícies metálicas, como as energias de adsorção envolvidas em processos catalíticos, os estados eletrônicos de vários metais, a sua influência na reatividade e o efeito de segregação superficial, presente em depósitos bimetálicos. Embora estes modelos ajudem na compreensão dos fenômenos que ocorrem em interfaces adsorbato-metal, até o presente momento a complexidade de tais procedimentos impede simulações que envolvam muitas partículas, que é caso de superfícies metálicas. Por conseguinte, as interações de longo alcance e as características de partículas que se encontram no seio de soluções metálicas têm que ser negligenciadas ou simplificadas, com perda de informação sobre a natureza e as causas de tais fenômenos.

\section{CONCLUSÃO}

O conhecimento dos fundamentos da eletrocatálise é primordial para que se possa controlar inúmeras reações de interesse prático. Neste trabalho foram enfatizadas duas reações, a RRO e ROH. Este par de reações rege o funcionamento de uma classe importante de células a combustível, as células do tipo PEMFC. Estudos realizados neste sistema demonstram que os principais fatores limitantes da corrente gerada são a baixa velocidade da RRO no lado catódico e a presença de impurezas adsorvidas no lado anódico. Em resposta a estas dificuldades, vários catalisadores à base de ligas ou codepósitos de Pt têm sido avaliados. Os dados eletroquímicos e o uso de técnicas de caracterização dos eletrocatalisadores têm permitido obter um panorama geral bem fundamentado da situação, de modo que atualmente se sabe que a presença de um segundo metal influencia sensivelmente a atividade catalítica da platina, seja pela geração de intermediários importantes para o mecanismo, seja pela modificação das propriedades estruturais e eletrônicas do catalisador.

$\mathrm{O}$ volume de informações obtido a partir destes esforços tem servido para ilustrar a complexidade cinética de tais reações, de forma que o uso de ferramentas experimentais e a elaboração de modelos teóricos ainda se fazem necessários para que se possa aprofundar o conhecimento acerca dos processos eletrocatalíticos.

\section{AGRADECIMENTOS}

Os autores agradecem à FAPESP, CNPq e CAPES pelos apoios recebidos.

\section{REFERÊNCIAS}

1. Ticianelli, E. A.; Gonzalez, E. R.; Quim. Nova 1989, 12, 268.

2. Wendt, H.; Linardi, M.; Aricó, E. M.; Quim. Nova 2002, 25, 470.

3. Wendt, H.; Götz, M.; Linardi, M.; Quim. Nova 2000, 23, 538.

4. Ticianelli, E. A.; Srinivasan, S.; Derouin, C. R.; Redondo, A.; J. Electrochem. Soc. 1988, 135, 2209.

5. Ticianelli, E. A.; Beery, J. G.; Srinivasan, S.; J. Appl. Electrochem. 1991, $21,597$.

6. Wilson, M. S.; Gottesfeld, S.; J. Appl. Electrochem. 1992, 22, 1.

7. Yeager, E. B.; Electrochim. Acta 1984, 29, 1527.

8. Yeager, E. B.; J. Mol. Catal. 1986, 38, 5.

9. Mukerjee, S.; Srivanasan, S.; Soriaga, M. P.; McBreen, J.; J. Electrochem. Soc. 1995, 142, 1409.

10. Min, M. K.; Cho, J. H.; Cho, K. W.; Kim, H.; Electrochim. Acta 2000, 45 4211.

11. Paulus, U. A.; Scherer, G. G.; Wokaun, A.; Schmidt, T. J.; Stamenkovic, V.; Radmilovic, V.; Markovic, N. M.; Ross, P. N.; J. Phys. Chem. B 2002 , 106,4181 .
12. Arai, H.; Muller, S.; Haas, O.; J. Electrochem. Soc. 2000, 147, 3584.

13. Kordech, K.; Hacker, V.; Gsellmann, J.; Cifrain, M.; Faleschini, G.; Enzinger, P.; Fankhauser, R.; Ortner, M.; Muhr, M.; Aronson, R. R.; J. Power Sources 2000, 86, 162.

14. Toda, T.; Igarashi, H.; Watanabe, M.; J. Electrochem. Soc. 1998, 145, 4185.

15. Jo, J. H.; Yi, S. C.; J. Power Sources 1999, 84, 87.

16. Tamizhmani, G.; Capuano, G. A.; J. Electrochem. Soc. 1994, 141, 968.

17. Toda, T.; Igarashi, H.; Uchida, H; Watanabe, M.; J. Electrochem. Soc. 1999, $146,3750$.

18. Paulus, U. A.; Wokaun, A.; Scherer, G. G.; Schmidt, T. J.; Stamenkovic, V.; Markovic, N. M.; Ross, P. N.; Electrochim. Acta 2002, 47, 3787.

19. Stamenkovic, V.; Schmidt, T. J.; Ross, P. N.; Markovic, N. M.; J. Electroanal.Chem. 2003, 554-555, 191.

20. Wang, J. X.; Markovic, N. M.; Adzic, R. R.; J. Phys. Chem. B 2004, 108, 4127.

21. Toda, T.; Igarashi, H.; Watanabe, M.; J. Electroanal. Chem. 1999, 460, 258.

22. Li, W.; Zhou, W.; Li, H.; Zhou, Z.; Zhou, B.; Sun, G.; Xin, Q.; Electrochim. Acta 2004, 49, 1045

23. Xiong, L.; Kannan, A. M.; Manthiram, A.; Electrochem. Commun. 2002, 4,898 .

24. Damjanovic, A.; Brusic, V.; Bockris, J. O. M.; J. Phys. Chem. 1967, 71, 2471 .

25. Adzic, R. R. Em Electrocatalysis; Lipkowski, J.; Ross, P. N., eds.; Ed. Wiley-VCH: New York, 1998, p. 209.

26. Jalan, V.; Taylor, J.; J. Electrochem. Soc. 1983, 130, 2299.

27. Appleby, A. J.; Energy 1986, 11, 13.

28. Mukerjee, S.; Srinivasan, S.; J. Electroanal. Chem. 1993, 357, 201.

29. Glass, J. T.; Cahen, G. L.; Stoner, G. E.; J. Electrochem. Soc. 1987, 134, 58.

30. Paffet, M. T.; Beery, G. J.; Gottesfeld, S.; J. Electrochem. Soc. 1998, 135, 1431.

31. Watanabe, M.; Tsurumi, K.; Mizukami, T.; Nakamura, T.; Stonehart, P.; J. Electrochem. Soc. 1994, 141, 2659.

32. Freund, A.; Lang, J.; Lehman, T.; Starz, K. A.; Catal. Today 1996, 27, 279.

33. Aricò, A. S.; Shukla, A. K.; Kim, H.; Park, S.; Min, M.; Antonucci,V.; Appl. Surf. Sci. 2001, 172, 33.

34. Antolini, E.; Passos, R. R.; Ticianelli, E. A.; Electrochim. Acta 2002, 48, 263.

35. Faubert, G.; Côté, R.; Guay, D.; Dodelet, J. P.; Dénès, G.; Poleunis, C.; Bertrand, P.; Electrochim. Acta 1998, 43, 1969.

36. Lin, W. F.; Iwasita, T.; Vielstich, W.; J. Phys. Chem. B 1999, 103, 3250.

37. Grgur, B. N.; Zhuang, G.; Markovic, N. M.; Ross, P. N.; J. Phys. Chem. B 1997, 101, 3910

38. Liu, R.; Iddir, H.; Fan. Q.; Hou, G.; Bo, A.; Ley, K. L.; Smotkin, E. S.; Sung, Y-E.; Kim, H.; Thomas, S.; Wieckowski, A.; J. Phys. Chem. B 2000, $104,3518$.

39. Wang, K.; Gasteiger, H. A.; Markovic, N. M.; Ross, P. N.; Electrochim. Acta 1996, 41, 2587.

40. Santiago, E. I.; Giz, M. J.; Ticianelli, E. A.; J. Solid State Electrochem. 2003, 7, 607 .

41. Mukerjee, S.; McBreen, J.; J. Electroanal. Chem. 1998, 448, 163.

42. Page, T.; Johnson, R.; Hormes, J.; Noding, S.; Rambabu, B.; J. Electroanal. Chem. 2000, 485, 34.

43. Camara, G. A.; Giz, M. J.; Paganin, V. A.; Ticianelli, E. A.; J. Electroanal. Chem. 2002, 537, 21.

44. Mukerjee, S.; Srinivasan, S.; Soriaga, M. P.; McBreen, J.; J. Phys. Chem. 1995, 99, 4577.

45. Mukerjee, S.; Urian, R. C.; Electrochim. Acta 2002, 47, 3219.

46. Mukerjee, S.; McBreen, J.; J. Electrochem. Soc. 1999, 146, 600.

47. Russell, A. E.; Maniguet, S.; Mathew, R. J.; Yao, J.; Roberts, M. A.; Thompsett, D.; J. Power Sources 2001, 96, 226.

48. Mukerjee, S.; Srinivasan, S.; Soriaga, M. P.; McBreen, J.; J. Electrochem. Soc. 1995, 142, 1409.

49. Liu, P.; Norskov, J. K.; Fuel Cells 2001, 1, 192

50. Mukerjee, S.; Lee, S. J.; Ticianelli, E. A.; McBreen, J.; Grgur, B. N.; Markovic, N. M.; Ross, P. N.; Giallombardo, J. R.; De Castro, E. S.; Electrochem. Solid-State Lett. 1999, 2, 12.

51. Ley, K. L.; Liu, R.; Pu, C.; Fan, Q.; Leyarovska, N.; Segre, C.; Smotkin, E. S.; J. Electrochem. Soc. 1997, 144, 1543.

52. Liu, P.; Norskov, J. K.; Phys. Chem. Chem. Phys. 2001, 3, 3814.

53. Norskov, J. K.; Bligaard, T.; Logadottir, A.; Van, S.; Hansen, L. B.; Bollinger, M.; Bengaard, H.; Hammer, B.; Sljivancanin, Z.; Mavrikakis, M.; Xu, Y.; Dahl, S.; Jacobsen, C. J. H.; J. Catal. 2002, 209, 275.

54. Hammer, B.; Norskov, J. K.; Surf. Sci. 1995, 343, 211.

55. Christoffensen, E.; Liu, P.; Ruban, A.; Skriver, H. L.; Norskov, J. K.; J. Catal. 2001, 199, 123. 